\title{
The (sub/super)additivity assertion of Choquet
}

\author{
by \\ HEINZ KÖNIG (Saarbrücken)
}

\begin{abstract}
The assertion in question comes from the short final section in Theory of capacities of Choquet (1953/54), in connection with his prototype of the subsequent Choquet integral. The problem was whether and when this operation is additive. Choquet had the much more abstract idea that all functionals in a certain wide class must be subadditive, and similarly for superadditivity. His treatment of this point was more like an outline, and his proof limited to a rather narrow special case. Thus the proper context and scope of the assertion has remained open. In this paper we present a counterexample which shows that the initial context has to be modified, and then in a new context we prove a comprehensive theorem which fulfils all the needs that have turned up so far.
\end{abstract}

In Section 48 of his famous Theory of capacities [2] Gustave Choquet introduced a certain class of functionals with the flavour of an integral, but invented for another purpose connected with capacities and not at all for the sake of measure and integration. Yet the concept showed basic qualities in that other respect too: It was in the initial spirit of Lebesgue [10] to construct the integral via decomposition into horizontal strips rather than vertical ones, which had fallen into oblivion in the course of the 20th century, and was simpler and much more comprehensive than the usual constructions. Thus in subsequent decades the concept developed into a universal one in measure and integration, called the Choquet integral. One could even wonder why the Choquet integral has not become the foundation for all of integration theory.

But the fact that this did not happen had an immediate reason: The basic hardship with the Choquet integral is that it is a priori obscure whether and when it is additive, which one subdivides into subadditive and superadditive. To this issue Choquet contributed in his final section 54 a spectacular, because much more abstract idea: On certain lattice cones all submodular

2000 Mathematics Subject Classification: 26A51, 26D15, 28A12, 28A25, 28C05, 28C15, $46 \mathrm{G} 12,52 \mathrm{~A} 40$.

Key words and phrases: (sub/super)modular and (sub/super)additive functionals, convex functions, Choquet integral, Stonean function classes, Stonean and truncable functionals, Daniell-Stone and Riesz representation theorems. 
and positive-homogeneous real-valued functionals must be subadditive, and the same for super in place of $s u b$. It is this assertion which forms the theme of the present paper (in what follows the two cases will be united via an obvious sub/super shorthand notation). The treatment of Choquet was more like an outline, and his proof limited to a rather narrow special case. While the Choquet integral has been explored in subsequent decades, the abstract assertion has remained unsettled up to now.

In recent years the present author needed an assertion of this kind to further develop his extended Daniell-Stone and Riesz representation theorems [7]. In 1998 he obtained an intermediate result which was sufficient for that purpose [8]. In this paper we present a counterexample which shows that the initial context for the abstract assertion has to be modified, and then in a new context we prove a comprehensive theorem which fulfils all the needs that have turned up so far. For the basic step in the so-called finite situation there will be two proofs. One of them is a distributional version of the initial proof due to Choquet, while the other one furnishes, via a remarkable fact on convex functions, an essential strengthening in the finite situation.

\section{INTRODUCTION AND FUNDAMENTALS}

The Choquet integral. The Choquet integral as evolved in the second half of the 20th century exists in different versions. The present version is from the author's textbook [7, Section 11]. It features two classes of admissible functions. The reason is that the two variants are in perfect accord with the two extension theories in measure and integration, the inner and outer one, developed in [7].

Let $X$ be a nonvoid set and $\mathfrak{S}$ be a lattice of subsets with $\emptyset \in \mathfrak{S}$ in $X$. We define $\operatorname{UM}(\mathfrak{S}) / \operatorname{LM}(\mathfrak{S})$ to consist of the functions $f \in[0, \infty]^{X}$ such that $[f \geq t] /[f>t] \in \mathfrak{S}$ for all $0<t<\infty$, called upper/lower measurable $\mathfrak{S}$. We fix an increasing set function $\varphi: \mathfrak{S} \rightarrow[0, \infty]$ with $\varphi(\emptyset)=0$ and define the Choquet integral

$$
\begin{array}{ll}
f f d \varphi:=\int_{0 \leftarrow}^{\rightarrow \infty} \varphi([f \geq t]) d t \in[0, \infty] \quad \text { for } f \in \mathrm{UM}(\mathfrak{S}), \\
f f d \varphi:=\int_{0 \leftarrow}^{\rightarrow \infty} \varphi([f>t]) d t \in[0, \infty] \quad \text { for } f \in \operatorname{LM}(\mathfrak{S}),
\end{array}
$$

both integrals being improper Riemann integrals of a monotone function with values in $[0, \infty]$. The definition is unambiguous since in case $f \in$ $\mathrm{UM}(\mathfrak{S}) \cap \operatorname{LM}(\mathfrak{S})$ the two second members are equal. Thus for $A \in \mathfrak{S}$ we have $\chi_{A} \in \mathrm{UM}(\mathfrak{S}) \cap \operatorname{LM}(\mathfrak{S})$ with $f \chi_{A} d \varphi=\varphi(A)$. If $\mathfrak{S}$ is a $\sigma$-algebra then 
$\operatorname{UM}(\mathfrak{S})=\operatorname{LM}(\mathfrak{S})$ consists of the functions $f \in[0, \infty]^{X}$ measurable $\mathfrak{S}$ in the usual sense, and if $\varphi$ is a measure then $f f d \varphi$ is the usual integral $\int f d \varphi$.

The prototype of the Choquet integral was defined in [2] for the lattice $\mathfrak{S}=\operatorname{Comp}(X)$ of compact subsets in a locally compact Hausdorff topological space $X$ and under the assumption $\varphi<\infty$, but restricted to the function class

$$
\mathrm{CK}\left(X,\left[0, \infty[) \subset \operatorname{USCK}\left(X,\left[0, \infty[) \subset \mathrm{UM}(\operatorname{Comp}(X)) \cap\left[0, \infty\left[{ }^{X},\right.\right.\right.\right.\right.\right.
$$

where the first two classes are defined to consist of the continuous and of the upper semicontinuous functions $X \rightarrow[0, \infty[$ with compact support. Therefore the set functions $\varphi$ had sometimes to be restricted to the downward $\tau$ continuous ones, that is, to the capacities in the sense of [2].

We return to the full Choquet integral. For the basic properties recorded below we refer to [7, Section 11] and [8, Section 2].

1.1. REMARK. For a function $f \in\left[0, \infty\left[{ }^{X}\right.\right.$ with finitely many values the following are equivalent.

(i) $f \in \operatorname{UM}(\mathfrak{S})$.

(ii) $f \in \operatorname{LM}(\mathfrak{S})$.

(iii) $f=\sum_{l=1}^{r} t_{l} \chi_{A(l)}$ for some real $t_{1}, \ldots, t_{r}>0$ and $A(1), \ldots, A(r) \in \mathfrak{S}$.

(iv) The same as (iii) with $A(1) \supset \ldots \supset A(r)$.

We define $\mathrm{S}(\mathfrak{S})$ to consist of the functions as in (i)-(iv); note from (iii) that $\mathrm{S}(\mathfrak{S})$ is stable under addition. For the representations (iv) of $f \in \mathrm{S}(\mathfrak{S})$ we have $f f d \varphi=\sum_{l=1}^{r} t_{l} \varphi(A(l))$.

1.2. Properties. (1) $\mathrm{UM}(\mathfrak{S})$ and $\mathrm{LM}(\mathfrak{S})$ are positive-homogeneous (under multiplication with real numbers $0<t<\infty$ ) with 0 and stable under pointwise maximum $\vee$ and pointwise minimum $\wedge$.

(2) If $\mathfrak{S}$ is stable under countable intersections then $\mathrm{UM}(\mathfrak{S})$ is stable under addition and $\mathrm{UM}(\mathfrak{S}) \supset \operatorname{LM}(\mathfrak{S})$. If $\mathfrak{S}$ is stable under countable unions then $\operatorname{LM}(\mathfrak{S})$ is stable under addition and $\operatorname{LM}(\mathfrak{S}) \supset \operatorname{UM}(\mathfrak{S})$.

(3) For an increasing $\varphi: \mathfrak{S} \rightarrow[0, \infty]$ with $\varphi(\emptyset)=0$ the Choquet integral $I(f)=f f d \varphi$ on $\mathrm{UM}(\mathfrak{S}) / \mathrm{LM}(\mathfrak{S})$ is positive-homogeneous and increasing under the pointwise order $\leq$.

The next point is important enough to be included, although it will not be needed later: The Beppo Levi theorem carries over to the Choquet integral, and in fact in most comprehensive sequential and nonsequential forms, provided that one adopts the integral in the version of [7]. We quote from $[7,11.17 / 18]$ in the obvious old notations.

1.3. ThEOREM. Let $\varphi: \mathfrak{S} \rightarrow[0, \infty]$ be increasing with $\varphi(\emptyset)=0$ and $I(f)=f f d \varphi$ on $\operatorname{UM}(\mathfrak{S}) / \operatorname{LM}(\mathfrak{S})$. For $\bullet=\sigma, \tau$ we have: 
(Inn) $\varphi$ is almost downward •-continuous $\Leftrightarrow I$ is almost downward •continuous on $\mathrm{UM}(\mathfrak{S})$.

(Out) $\varphi$ is upward $\bullet$-continuous $\Leftrightarrow I$ is upward $\bullet$-continuous on $\operatorname{LM}(\mathfrak{S})$.

In the present context the basic question is when the Choquet integral $I(f)=f f d \varphi$ on $\operatorname{UM}(\mathfrak{S}) / \operatorname{LM}(\mathfrak{S})$ is (sub/super)additive, and also when it is (sub/super)modular. A functional $I: S \rightarrow[0, \infty]$ on a nonvoid function system $S \subset[0, \infty]^{X}$ is defined to be (sub/super) additive if

$$
I(u+v) \leq / \geq I(u)+I(v) \text { for all } u, v \in S \text { such that } u+v \in S,
$$

so that $S$ need not be stable under addition; if $S$ is stable under $\vee \wedge$, we define $I$ to be (sub/super) modular (under $\vee \wedge$, for short $\vee \wedge$ ) if

$$
I(u \vee v)+I(u \wedge v) \leq / \geq I(u)+I(v) \quad \text { for all } u, v \in S .
$$

In the case of the Choquet integral $I$ these properties will of course be related to the behaviour of the set function $\varphi$. A set function on a lattice is defined to be (sub/super) modular if

$$
\varphi(A \cup B)+\varphi(A \cap B) \leq / \geq \varphi(A)+\varphi(B) \quad \text { for all } A, B \in \mathfrak{S} .
$$

We note two simple observations.

1.4. REMARK. The Choquet integral I on $\operatorname{UM}(\mathfrak{S}) / \operatorname{LM}(\mathfrak{S})$ satisfies:

(A) $\varphi($ sub/super $)$ modular $\Leftarrow I$ (sub/super $)$ additive,

(M) $\varphi$ (sub/super) modular $\Leftrightarrow I$ (sub/super) modular.

Proof. One obtains the two $\Leftarrow$ implications from $(A, B \in \mathfrak{S})$

$$
\begin{aligned}
I\left(\chi_{A}+\chi_{B}\right)=I\left(\chi_{A \cup B}+\chi_{A \cap B}\right) & =\varphi(A \cup B)+\varphi(A \cap B), \\
I\left(\chi_{A} \vee \chi_{B}\right)+I\left(\chi_{A} \wedge \chi_{B}\right)=I\left(\chi_{A \cup B}\right)+I\left(\chi_{A \cap B}\right) & =\varphi(A \cup B)+\varphi(A \cap B),
\end{aligned}
$$

the first of which follows from 1.1, both combined with $I\left(\chi_{A}\right)+I\left(\chi_{B}\right)=$ $\varphi(A)+\varphi(B)$. To obtain $\Rightarrow$ in $(\mathrm{M})$ one notes that for $u, v \in \mathrm{UM}(\mathfrak{S})$,

$$
\begin{aligned}
I(u \vee v)+I(u \wedge v) & =\int_{0 \leftarrow \infty}^{\rightarrow \infty}(\varphi([u \vee v \geq t])+\varphi([u \wedge v \geq t])) d t \\
& \left.=\int_{0 \leftarrow \infty}^{\rightarrow \infty}(\varphi([u \geq t] \cup[v \geq t])+\varphi([u \geq t]) \cap[u \geq t])\right) d t \\
& \leq / \geq \int_{0 \leftarrow}^{\rightarrow \infty}((\varphi([u \geq t])+\varphi([v \geq t])) d t=I(u)+I(v),
\end{aligned}
$$

and the same on $\operatorname{LM}(\mathfrak{S})$.

We note that for the prototype in [2] with its restricted domain the two $\Leftarrow$ implications require that $\varphi$ be a capacity. The decisive question is whether $\Rightarrow$ holds true in (A). It will be dealt with in what follows. 
The work of Choquet (1953/54). Choquet [2] noted that for his prototype the $\Rightarrow$ implication in (A) holds true, and hence that the three properties involved in $(\mathrm{A}) \&(\mathrm{M})$ are equivalent for capacities $\varphi$. But what is more and deserves to be called spectacular, he had the idea that the implication

$$
I \text { (sub/super)modular } \Rightarrow I \text { (sub/super)additive }
$$

must be valid for a much wider class of functionals (he also knew that this cannot be true for the converse implication). His precise formulation 54.1 was as follows.

1.5. Choquet's Vision. Let $E$ be an ordered vector space with order $\sqsubseteq$ and positive cone $E^{+}$, and assume that $E$ (or at least $E^{+}$) is a lattice under $\sqsubseteq$ with lattice operations $\sqcup \sqcap$. Let $I: E^{+} \rightarrow \mathbb{R}$ be positive-homogeneous. If I is (sub/super) modular under $\sqcup \sqcap$ then it must be (sub/super) additive.

From 1.5 applied to $E=\mathrm{CK}(X, \mathbb{R})$ on the locally compact Hausdorff $X$ with pointwise order $\leq$ and lattice operations $\vee \wedge$, and to the restricted Choquet integral $I(f)=f f d \varphi$ on $E^{+}=\operatorname{CK}(X,[0, \infty[)$ with an arbitrary $\varphi$, and combined with $\Rightarrow$ in $(M)$, it follows indeed that Choquet's prototype satisfies the desired implication $\Rightarrow$ in $(\mathrm{A})$.

However, Choquet did not prove 1.5 in its full extent. His proof was restricted to the case $E=\mathbb{R}^{n}$ with pointwise order $\leq$ and lattice operations $\vee \wedge$, and to positive-homogeneous functions $I: E^{+}=\left[0, \infty\left[^{n} \rightarrow \mathbb{R}\right.\right.$ which are continuous on $\left[0, \infty\left[^{n}\right.\right.$ and $\mathrm{C}^{2}$ on $] 0, \infty{ }^{n}$. The explanation is that the entire context was at the end and outside the mainstream of the memoir [2]. Nevertheless Choquet's proof was so well-founded that after half a century it is capable, as we shall see, to furnish a proof of the basic step for the present new main theorem.

The need for reconsideration. We have seen that Choquet's vision 1.5 furnishes the decisive implication $\Rightarrow$ in $(\mathrm{A})$ for his prototype. But we have to note that it does not furnish this implication for the full Choquet integral. An obvious reason is that the functions $f \in \mathrm{UM}(\mathfrak{S}) / \mathrm{LM}(\mathfrak{S})$ and the functional $I(f)=f f d \varphi$ can attain the value $\infty$, and another one that the domains $\operatorname{UM}(\mathfrak{S}) / \operatorname{LM}(\mathfrak{S})$ need not be stable under addition. But there is a deeper reason: Let for example $X=[0,1]$ and $\mathfrak{S}=\operatorname{Comp}(X)$. Then $\operatorname{USC}\left(X,\left[0, \infty[)=\operatorname{UM}(\mathfrak{S}) \cap\left[0, \infty\left[^{X}\right.\right.\right.\right.$ is a convex cone in the vector space $E=\mathrm{B}(X, \mathbb{R})$ of bounded functions, pointed and salient in the usual sense and hence the positive cone $E^{+} \subset E$ in the so-called intrinsic order $\sqsubseteq$ on $E$ which it produces. We shall prove that this $E^{+}$is not a lattice under $\sqsubseteq$. Thus 1.5 cannot be applied.

1.6. Remark. On $X=[0,1]$ the convex cone $S=\operatorname{USC}(X,[0, \infty[) \subset$ $\mathrm{B}(X, \mathbb{R})$ is not a lattice in its intrinsic order $\sqsubseteq$. 
Proof. We fix $0<a<b<1$ and form $u=\chi_{[0, b]}$ and $v=\chi_{[a, 1]}$ in $S$. We claim that there is no $w \in S$ such that

(i) $u, v \sqsubseteq w$, and

(ii) each $h \in S$ with $u, v \sqsubseteq h$ satisfies $w \sqsubseteq h$.

To see this we form $f=1+\chi_{\{a, b\}}$ and $g=1+\chi_{[a, b]}$ in $S$. We check three little facts.

(0) $(h \in S$ with $u, v \sqsubseteq h) \Rightarrow f \leq h$. In fact, we have $u, v \leq h$ and hence need to show that $h(a), h(b) \geq 2$. Now for instance $h-u \in S$ and ]$b, 1] \subset[h-u \geq 1]$ and hence $[b, 1] \subset[h-u \geq 1]$, so that $h(b) \geq 2$.

(1) $u, v \sqsubseteq f$. In fact, for instance $f-u=\chi_{] b, 1]}+\chi_{\{a, b\}}=\chi_{[b, 1]}+\chi_{\{a\}} \in S$.

(2) $u, v \sqsubseteq g$. In fact, we have $g=u+v$ and thus for instance $g-u=$ $v \in S$.

Now assume that $w \in S$ satisfies (i)-(ii). Then

- (i) and (0) applied to $h=w$ yield $f \leq w$,

- (1) and (ii) applied to $h=f$ yield $w \sqsubseteq f$,

- (2) and (ii) applied to $h=g$ yield $w \sqsubseteq g$,

which combine to $f=w \sqsubseteq g$. But this is false since $g-f=\chi_{] a, b[} \notin S$.

To be sure, the $\Rightarrow$ implication in (A) holds true for the full Choquet integral, even though this does not follow from 1.5. In the second half of the 20th century the implication was proved a number of times for different versions of the Choquet integral. As far as the author is aware, the first (1974) proof is due to Topsøe [12]. For other proofs see [1], [6], [11], [3]. The implication for the present version is in [7, 11.11].

On the other hand Choquet's vision 1.5 was left open in [2], and in fact remained open until March 2002, when the present author observed that the statement is not true as it stands.

1.7. Example. Let $E=\mathbb{R}^{2}$ be equipped with the lexicographical order $\sqsubseteq$, that is, $u=\left(u_{1}, u_{2}\right)$ and $v=\left(v_{1}, v_{2}\right)$ satisfy $u \sqsubseteq v$ iff either $u_{1}<v_{1}$, or $u_{1}=v_{1}$ and $u_{2} \leq v_{2}$. The order $\sqsubseteq$ is compatible with the standard vector space structure, and $E^{+}$consists of the halfspace $\left\{x: x_{1}>0\right\}$ and the halfline $\left\{x: x_{1}=0\right.$ and $\left.x_{2} \geq 0\right\}$. Moreover $\sqsubseteq$ is total and hence a lattice order with trivial lattice operations $\sqcup П$. Thus in particular all positive-homogeneous functions $I: E^{+} \rightarrow \mathbb{R}$ are modular $\sqcup \sqcap$. But of course most of them are not additive. A simple example is $I(x)=x_{2}^{+}$, since $u=\left(u_{1}, u_{2}\right)$ and $v=\left(v_{1}, v_{2}\right)$ with $u_{1}, v_{1}>0$ and $u_{2}<0<v_{2}$ are in $E^{+}$and satisfy $I(u+v)=\left(u_{2}+v_{2}\right)^{+}<v_{2}=v_{2}^{+}=I(v)=I(u)+I(v)$.

The same idea works for any real vector space $E$ of dimension $>1$, in that one defines a compatible and total order $\sqsubseteq$ on $E$ via the choice of a basis $B$ of $E$ and of a well-order of $B$. 
Thus there is some reason for reconsideration. This does not mean to question the wonderful overall implication

$$
I \text { (sub/super)modular } \Rightarrow I \text { (sub/super)additive, }
$$

which will henceforth be called the fundamental implication. In what follows the author wants to develop what he has observed since 1998. Section 2 will be devoted to the special case $E=\mathbb{R}^{n}$ with pointwise order $\leq$ and lattice operations $\vee \wedge$, henceforth called the finite situation, and Section 3 to the full situation, as we shall see with pointwise order and lattice operations as well. It will be concluded with an application to the Daniell-Stone and Riesz representation theorems mentioned in the introduction.

\section{THE FINITE SITUATION}

The basic step. The basic step is the result which follows. It is due to Choquet [2] under the assumption that the function $I$ is $\mathrm{C}^{2}$. We first sketch his proof.

2.1. Proposition. Assume that $I:] 0, \infty\left[{ }^{n} \rightarrow \mathbb{R}\right.$ is positive-homogeneous and continuous. Then I satisfies the fundamental implication.

Sketch of proof (in case $I$ is $\mathrm{C}^{2}$ ). Let $\left.X_{1}, \ldots, X_{n}:\right] 0, \infty\left[^{n} \rightarrow\right] 0, \infty[$ denote the coordinate functions and $D_{1}, \ldots, D_{n}$ the partial derivations. One verifies three facts:

(0) For $z=\left(z_{1}, \ldots, z_{n}\right) \in \mathbb{R}^{n}$ one has the identity

$$
\begin{aligned}
\sum_{k, l=1}^{n} z_{k} z_{l}\left(D_{k} D_{l} I\right)= & \sum_{k=1}^{n} \frac{z_{k}^{2}}{X_{k}}\left(\sum_{l=1}^{n} X_{l}\left(D_{k} D_{l} I\right)\right) \\
& -\frac{1}{2} \sum_{k, l=1}^{n} X_{k} X_{l}\left(\frac{z_{k}}{X_{k}}-\frac{z_{l}}{X_{l}}\right)^{2}\left(D_{k} D_{l} I\right) .
\end{aligned}
$$

(1) If $I$ is positive-homogeneous then $\sum_{l=1}^{n} X_{l}\left(D_{k} D_{l} I\right)=0$ for $1 \leq k \leq n$.

(2) If $I$ is (sub/super)modular $\vee \wedge$ then $D_{k} D_{l} I \leq / \geq 0$ for $1 \leq k \neq l \leq n$.

For $I$ positive-homogeneous and (sub/super)modular $\vee \wedge$ these facts combine with Taylor's formula to furnish that $I$ is convex/concave, hence (sub/super)additive.

Below we present two proofs of 2.1. The first proof extends Choquet's partial result via distribution theory. This has not been done before, perhaps because one tried to extend the result via regularization, which does not work. By contrast we shall follow Choquet's proof, in that we take the above steps (0)-(2) in the distributional sense. 
First proof of 2.1. We put $U:=] 0, \infty\left[{ }^{n} \subset \mathbb{R}^{n}\right.$. Let as above $X_{1}, \ldots, X_{n}$ : $U \rightarrow] 0, \infty\left[\right.$ denote the coordinate functions $x=\left(x_{1}, \ldots, x_{n}\right) \mapsto x_{1}, \ldots, x_{n}$, and $D_{1}, \ldots, D_{n}$ the partial derivations in both the standard and distributional sense. Moreover let $\nabla(u, \delta)$ denote the closed ball of radius $\delta$ around $u$. Assume that $I: U \rightarrow \mathbb{R}$ is continuous.

(1) If $I$ is positive-homogeneous then

$$
A_{k}:=\sum_{l=1}^{n} X_{l}\left(D_{k} D_{l} I\right)=0 \quad \text { for } 1 \leq k \leq n .
$$

In fact, for $\varphi \in \mathrm{CK}^{\infty}(U)$ we have in the usual notations

$$
\left\langle A_{k}, \varphi\right\rangle=\left\langle I, \sum_{l=1}^{n} D_{k} D_{l}\left(X_{l} \varphi\right)\right\rangle=\left\langle I,(n+1) D_{k} \varphi+\sum_{l=1}^{n} X_{l}\left(D_{l} D_{k} \varphi\right)\right\rangle .
$$

For $\mathrm{S}^{n} \subset \mathbb{R}^{n}$ the unit sphere and $\sigma^{n}$ the surface measure on $\mathrm{S}^{n}$ we obtain

$$
\left\langle A_{k}, \varphi\right\rangle=\int_{\mathrm{S}^{n} \cap U} \int_{0 \leftarrow}^{\rightarrow \infty} t^{n-1} I(t s)\left((n+1) D_{k} \varphi(t s)+\sum_{l=1}^{n} t s_{l} D_{l} D_{k} \varphi(t s)\right) d t d \sigma^{n}(s) .
$$

With $\varphi_{s} \in \mathrm{CK}^{\infty}(] 0, \infty[)$ for $s \in \mathrm{S}^{n}$ defined by $\varphi_{s}(t)=D_{k} \varphi(t s)$ it follows that

$$
\begin{aligned}
\left\langle A_{k}, \varphi\right\rangle & =\int_{\mathrm{S}^{n} \cap U} I(s)\left(\int_{0 \leftarrow}^{\rightarrow \infty}\left((n+1) t^{n} \varphi_{s}(t)+t^{n+1} \varphi_{s}^{\prime}(t)\right) d t\right) d \sigma^{n}(s) \\
& =\int_{\mathrm{S}^{n} \cap U} I(s)\left(\int_{0 \leftarrow}^{\rightarrow \infty}\left(t^{n+1} \varphi_{s}(t)\right)^{\prime} d t\right) d \sigma^{n}(s)=0 .
\end{aligned}
$$

(2) If $I$ is (sub/super)modular $\vee \wedge$ then

$$
D_{k} D_{l} I \leq / \geq 0 \quad \text { for } 1 \leq k \neq l \leq n \text { in the distributional sense, }
$$

that is, $\left\langle D_{k} D_{l} I, \varphi\right\rangle \leq / \geq 0$ for all $0 \leq \varphi \in \mathrm{CK}^{\infty}(U)$. For the proof fix $0 \leq \varphi \in \mathrm{CK}^{\infty}(U)$ and real $s, t>0$ so small that $\operatorname{supp}(\varphi)+\nabla(0, s+t) \subset U$. Then in the usual notations

$$
\begin{aligned}
\int_{U} I & (x)\left(\varphi\left(x-s e^{k}-t e^{l}\right)+\varphi(x)-\varphi\left(x-s e^{k}\right)-\varphi\left(x-t e^{l}\right)\right) d L(x) \\
= & \int_{U}\left(I\left(x+s e^{k}+t e^{l}\right)+I(x)-I\left(x+s e^{k}\right)-I\left(x+t e^{l}\right)\right) \varphi(x) d L(x) \\
= & \int_{U}\left(I\left(\left(x+s e^{k}\right) \vee\left(x+t e^{l}\right)\right)+I\left(\left(x+s e^{k}\right) \wedge\left(x+t e^{l}\right)\right)\right. \\
& \left.\quad-I\left(x+s e^{k}\right)-I\left(x+t e^{l}\right)\right) \varphi(x) d L(x) \leq / \geq 0 .
\end{aligned}
$$


After multiplication with $1 / s$ and letting $s \downarrow 0$ we obtain

$$
\int_{U} I(x)\left(-D_{k} \varphi\left(x-t e^{l}\right)+D_{k} \varphi(x)\right) d L(x) \leq / \geq 0,
$$

and then after multiplication with $1 / t$ and letting $t \downarrow 0$ we finally get

$$
\left\langle D_{k} D_{l} I, \varphi\right\rangle=\int_{U} I(x) D_{l} D_{k} \varphi(x) d L(x) \leq / \geq 0 .
$$

(3) The final step uses the Taylor formula of second degree with remainder term in integral form, which for $f \in \mathrm{CK}^{2}(U)$ reads

$$
f(u+x)=f(u)+\sum_{l=1}^{n} x_{l} D_{l} f(u)+\sum_{k, l=1}^{n} x_{k} x_{l} \int_{0}^{1} D_{k} D_{l} f(u+t x)(1-t) d t
$$

for $u, u+x \in U$. We fix $a, b \in U$ and put $u=\frac{1}{2}(a+b)$, so that $u-a=z$ and $u-b=-z$ with $z=\frac{1}{2}(b-a)$. Also fix $\delta>0$ with $|a, b|+\nabla(0, \delta) \subset U$, where $|a, b|$ denotes the line segment between $a$ and $b$. Then for $0 \leq \varphi \in \mathrm{CK}^{\infty}\left(\mathbb{R}^{n}\right)$ with $\operatorname{supp}(\varphi) \subset \nabla(u, \delta)$ consider

$$
\begin{aligned}
& A(\varphi):=\int_{\nabla(u, \delta)}(I(a-u+x)+I(b-u+x)-2 I(x)) \varphi(x) d L(x) \\
& =\int_{U} I(x)((\varphi(x+z)-\varphi(x))+(\varphi(x-z)-\varphi(x))) d L(x) \\
& =\int_{U} I(x)\left(\sum_{k, l=1}^{n} z_{k} z_{l} \int_{0}^{1}\left(D_{k} D_{l} \varphi(x+s z)+D_{k} D_{l} \varphi(x-s z)\right)(1-s) d s\right) d L(x) \\
& \quad=\int_{0}^{1}(1-s)\left\langle\sum_{k, l=1}^{n} z_{k} z_{l}\left(D_{k} D_{l} I\right), \varphi(\cdot+s z)+\varphi(\cdot-s z)\right\rangle d s .
\end{aligned}
$$

Now one has as in (0) above the relation

$$
\begin{aligned}
\sum_{k, l=1}^{n} z_{k} z_{l}\left(D_{k} D_{l} I\right)= & \sum_{k=1}^{n} \frac{z_{k}^{2}}{X_{k}}\left(\sum_{l=1}^{n} X_{l}\left(D_{k} D_{l} I\right)\right) \\
& -\frac{1}{2} \sum_{k, l=1}^{n} X_{k} X_{l}\left(\frac{z_{k}}{X_{k}}-\frac{z_{l}}{X_{l}}\right)^{2}\left(D_{k} D_{l} I\right) .
\end{aligned}
$$

For $I$ positive-homogeneous and (sub/super)modular $\vee \wedge$ this relation implies in view of (1)-(2) that

$$
-\sum_{k, l=1}^{n} z_{k} z_{l}\left(D_{k} D_{l} I\right) \leq / \geq 0 \quad \text { in the distributional sense. }
$$


It follows that $-A(\varphi) \leq / \geq 0$ for all functions $\varphi$ under consideration. Therefore $2 I(x) \leq / \geq I(a-u+x)+I(b-u+x)$ for all $x \in \nabla(u, \delta)$, since $I$ is continuous. In particular for $x=u$ we have $I(a+b)=2 I(u) \leq / \geq I(a)+I(b)$.

An important specialization of 2.1 is the case where $I$ is increasing under $\leq$ (also called isotone), because it is the unique one which will reach the full situation, but on the other hand will cover all applications known so far.

2.2. Specialization. Assume that $I:] 0, \infty\left[^{n} \rightarrow \mathbb{R}\right.$ is positive-homogeneous and increasing. Then $I$ is $\geq 0$ and continuous. Thus $I$ satisfies the fundamental implication.

Proof. (1) For $x \in] 0, \infty\left[{ }^{n}\right.$ we have $I(x) \leq I(2 x)=2 I(x)$ and hence $I(x) \geq 0$.

(2) If $a \in] 0, \infty\left[{ }^{n}\right.$ and $0<\varepsilon<1$ then $\{x:(1-\varepsilon) a \leq x \leq(1+\varepsilon) a\}$ is a neighbourhood of $a$ on which $(1-\varepsilon) I(a)=I((1-\varepsilon) a) \leq I(x) \leq I((1+\varepsilon) a)=$ $(1+\varepsilon) I(a)$, that is, $|I(x)-I(a)| \leq \varepsilon I(a)$.

Results on convex functions. The second proof of the basic step 2.1 will be based on certain facts on convex functions, and will lead to stronger versions. We state and prove our results for convex functions on convex subsets of real vector spaces instead of intervals in $\mathbb{R}$, because this requires no further effort and is the form in which the results will be needed. The first result is a mild strengthening of [5, Theorem 88], and is included for the sake of completeness. Note that [5] has a different definition of convex functions.

2.3. Remark. Let $K \subset E$ be a nonvoid convex subset of the real vector space $E$ and $f: K \rightarrow \mathbb{R}$. Assume that:

(i) for each pair $u, v \in K$ there exists $0<t<1$ such that

$$
f((1-t) u+t v) \leq(1-t) f(u)+t f(v) ;
$$

(ii) for each pair $u, v \in K$ the function $t \mapsto f((1-t) u+t v)$ is continuous on $0<t<1$.

Then $f$ is convex.

Proof. Assume not. Then there exist $u, v \in K$ such that

$$
M:=\{0<t<1: f((1-t) u+t v)>(1-t) f(u)+t f(v)\} \neq \emptyset .
$$

It follows from (ii) that $M \subset] 0,1[$ is open. Let $T \subset M$ be one of its connected components, that is, $T=] \alpha, \beta[$ with $0 \leq \alpha<\beta \leq 1$. Then

$$
\begin{aligned}
& f((1-\alpha) u+\alpha v) \leq(1-\alpha) f(u)+\alpha f(v), \\
& f((1-\beta) u+\beta v) \leq(1-\beta) f(u)+\beta f(v) .
\end{aligned}
$$

In fact, the first assertion is obvious when $\alpha=0$, and for $0<\alpha<1$ as well 
since $\alpha \notin M$. Now from (i) we have an $0<s<1$ such that

$$
\begin{aligned}
f((1-s)( & (1-\alpha) u+\alpha v)+s((1-\beta) u+\beta v)) \\
& \leq(1-s) f((1-\alpha) u+\alpha v)+s f((1-\beta) u+\beta v) \\
& \leq(1-s)((1-\alpha) f(u)+\alpha f(v))+s((1-\beta) f(u)+\beta f(v)) .
\end{aligned}
$$

This means that for $t:=(1-s) \alpha+s \beta$ we have $f((1-t) u+t v) \leq(1-t) f(u)+$ $t f(v)$, which contradicts the fact that $\alpha<t<\beta$ and hence $t \in M$.

The next result is much harder. The author must admit that he does not understand in full what is behind it. The special case $\varphi=$ const is [5, Theorem 111].

2.4. THEOREM. Let $K \subset E$ be a nonvoid convex subset of the real vector space $E$ and $f: K \rightarrow \mathbb{R}$. Assume that:

(i) there exists an affine function $\varphi: K \rightarrow] 0, \infty[$ such that

$$
f\left(\frac{\sqrt{\varphi(v)} u+\sqrt{\varphi(u)} v}{\sqrt{\varphi(v)}+\sqrt{\varphi(u)}}\right) \leq \frac{\sqrt{\varphi(v)} f(u)+\sqrt{\varphi(u)} f(v)}{\sqrt{\varphi(v)}+\sqrt{\varphi(u)}} \quad \text { for } u, v \in K
$$

(ii) for each pair $u, v \in K$ the function $t \mapsto f((1-t) u+t v)$ is bounded above on some nondegenerate subinterval of $\{t \in \mathbb{R}:(1-t) u+t v \in K\}$.

Then $f$ is convex.

We start with a technical lemma.

2.5. Lemma. For $t \in \mathbb{R}$ define $h_{t}:[0,1] \rightarrow \mathbb{R}$ by

$$
h_{t}(s)= \begin{cases}s & \text { for } t=0 \\ \frac{e^{t s}-1}{e^{t}-1} & \text { for } t \neq 0\end{cases}
$$

Then:

(1) $1-h_{t}(s)+h_{t}(s) e^{t}=e^{t s}$.

(2) $h_{t}$ is $\mathrm{C}^{1}$ with $e^{-|t|} \leq h_{t}^{\prime}(s) \leq e^{|t|}$ and hence strictly increasing. Moreover $h_{t}(0)=0$ and $h_{t}(1)=1$, so that $h_{t}$ is a bijection of $[0,1]$.

(3) For $\alpha, \beta \in[0,1]$ we have

$$
h_{t}\left(\frac{\alpha+\beta}{2}\right)=\frac{e^{t \beta / 2}}{e^{t \beta / 2}+e^{t \alpha / 2}} h_{t}(\alpha)+\frac{e^{t \alpha / 2}}{e^{t \beta / 2}+e^{t \alpha / 2}} h_{t}(\beta) .
$$

Proof of 2.5. (2) $h_{t}$ is $\mathrm{C}^{1}$ with $h_{t}^{\prime}=\frac{t}{e^{t}-1} e^{t s}$ in case $t \neq 0$. The estimate then follows from $x \leq e^{x}-1 \leq x e^{x}$ for $x \in \mathbb{R}$.

(3) In case $t \neq 0$ we have

$$
\begin{aligned}
h_{t}\left(\frac{\alpha+\beta}{2}\right)\left(e^{t}-1\right)\left(e^{t \beta / 2}+e^{t \alpha / 2}\right) & =\left(e^{t \beta / 2}+e^{t \alpha / 2}\right)\left(e^{t \alpha / 2} e^{t \beta / 2}-1\right) \\
& =e^{t \beta / 2}\left(e^{t \alpha}-1\right)+e^{t \alpha / 2}\left(e^{t \beta}-1\right),
\end{aligned}
$$

and hence the assertion. 
Now we subdivide the proof of 2.4 into four parts. For each part we fix $u, v \in K$ and put $t=\log \frac{\varphi(v)}{\varphi(u)}$, so that $\varphi(v)=e^{t} \varphi(u)$, and take $h_{t}:[0,1]$ $\rightarrow[0,1]$ as defined in 2.5.

PART 1. Define

$$
\begin{aligned}
M:=\left\{s \in[0,1]: f\left(\left(1-h_{t}(s)\right)\right.\right. & \left.u+h_{t}(s) v\right) \\
& \left.\leq\left(1-h_{t}(s)\right) f(u)+h_{t}(s) f(v)\right\} \subset[0,1] .
\end{aligned}
$$

Thus $0,1 \in M$. Then $\alpha, \beta \in M \Rightarrow(\alpha+\beta) / 2 \in M$. Hence $M$ contains all dyadic rationals $s \in[0,1]$.

Proof. Fix $\alpha, \beta \in[0,1]$ and put

$$
\begin{aligned}
a & =\left(1-h_{t}(\alpha)\right) u+h_{t}(\alpha) v, \\
b & =\left(1-h_{t}(\beta)\right) u+h_{t}(\beta) v, \\
c & =\left(1-h_{t}\left(\frac{\alpha+\beta}{2}\right)\right) u+h_{t}\left(\frac{\alpha+\beta}{2}\right) v .
\end{aligned}
$$

Then 2.5(3) yields

$$
c=\frac{e^{t \beta / 2}}{e^{t \beta / 2}+e^{t \alpha / 2}} a+\frac{e^{t \alpha / 2}}{e^{t \beta / 2}+e^{t \alpha / 2}} b .
$$

On the other hand 2.5(1) implies that $\varphi(a)=e^{t \alpha} \varphi(u)$ and $\varphi(b)=e^{t \beta} \varphi(u)$, and hence

$$
\frac{e^{t \beta / 2}}{e^{t \beta / 2}+e^{t \alpha / 2}}=\frac{\sqrt{\varphi(b)}}{\sqrt{\varphi(b)}+\sqrt{\varphi(a)}}, \quad \frac{e^{t \alpha / 2}}{e^{t \beta / 2}+e^{t \alpha / 2}}=\frac{\sqrt{\varphi(a)}}{\sqrt{\varphi(b)}+\sqrt{\varphi(a)}} .
$$

Thus by assumption (i) we have

$$
f(c) \leq \frac{e^{t \beta / 2}}{e^{t \beta / 2}+e^{t \alpha / 2}} f(a)+\frac{e^{t \alpha / 2}}{e^{t \beta / 2}+e^{t \alpha / 2}} f(b) .
$$

Now assume that $\alpha, \beta \in M$, that is,

$$
\begin{aligned}
& f(a) \leq\left(1-h_{t}(\alpha)\right) f(u)+h_{t}(\alpha) f(v), \\
& f(b) \leq\left(1-h_{t}(\beta)\right) f(u)+h_{t}(\beta) f(v) .
\end{aligned}
$$

Then these inequalities combine with $2.5(3)$ to furnish

$$
f(c) \leq\left(1-h_{t}\left(\frac{\alpha+\beta}{2}\right)\right) f(u)+h_{t}\left(\frac{\alpha+\beta}{2}\right) f(v)
$$

which means that $(\alpha+\beta) / 2 \in M$.

PART 2. Fix $0 \leq \alpha<\beta \leq 1$ and set as before

$$
a=\left(1-h_{t}(\alpha)\right) u+h_{t}(\alpha) v, \quad b=\left(1-h_{t}(\beta)\right) u+h_{t}(\beta) v .
$$

For these $a, b \in K$ put $\tau=\log \frac{\varphi(b)}{\varphi(a)}$, so that $\varphi(b)=e^{\tau} \varphi(a)$, and take $h_{\tau}$ as in 2.5. Then: 
(1) $\tau=t(\beta-\alpha)$.

(2) For $\alpha \leq s \leq \beta$ we have

$$
\left(1-h_{t}(s)\right) u+h_{t}(s) v=\left(1-h_{\tau}(\sigma)\right) a+h_{\tau}(\sigma) b \quad \text { with } \quad \sigma=\frac{s-\alpha}{\beta-\alpha} .
$$

Proof. (1) follows from $\varphi(a)=e^{t \alpha} \varphi(u)$ and $\varphi(b)=e^{t \beta} \varphi(u)$.

(2) From (1) we have $\tau \sigma=t(s-\alpha)$. Then

$$
\begin{aligned}
& \left(1-h_{\tau}(\sigma)\right) a+h_{\tau}(\sigma) b \\
& =\left(1-h_{\tau}(\sigma)\right)\left(\left(1-h_{t}(\alpha)\right) u+h_{t}(\alpha) v\right)+h_{\tau}(\sigma)\left(\left(1-h_{t}(\beta)\right) u+h_{t}(\beta) v\right) \\
& =(1-\zeta) u+\zeta v \quad \text { with } \quad \zeta=\left(1-h_{\tau}(\sigma)\right) h_{t}(\alpha)+h_{\tau}(\sigma) h_{t}(\beta) .
\end{aligned}
$$

In case $t=0$ (and hence $\tau=0$ ) we have

$$
\zeta=(1-\sigma) \alpha+\sigma \beta=\frac{\beta-s}{\beta-\alpha} \alpha+\frac{s-\alpha}{\beta-\alpha} \beta=s,
$$

and in case $t \neq 0$ (and hence $\tau \neq 0$ ) we have

$$
\begin{aligned}
\left(e^{t}-1\right)\left(e^{\tau}-1\right) \zeta & =\left(e^{\tau}-e^{\tau \sigma}\right)\left(e^{t \alpha}-1\right)+\left(e^{\tau \sigma}-1\right)\left(e^{t \beta}-1\right) \\
& =\left(e^{t(\beta-\alpha)}-e^{t(s-\alpha)}\right)\left(e^{t \alpha}-1\right)+\left(e^{t(s-\alpha)}-1\right)\left(e^{t \beta}-1\right) \\
& =\left(e^{t s}-1\right)\left(e^{t(\beta-\alpha)}-1\right)=\left(e^{t s}-1\right)\left(e^{\tau}-1\right),
\end{aligned}
$$

that is, $\zeta=h_{t}(s)$.

PART 3. The function $\lambda \mapsto f((1-\lambda) u+\lambda v)$ is bounded above for $0 \leq$ $\lambda \leq 1$.

Proof. (1) By assumption (ii), for fixed $u, v \in K$ we have real $\alpha<\beta$ such that

$$
(1-t) u+t v \in K \quad \text { and } \quad f((1-t) u+t v) \leq c<\infty \quad \text { for } \alpha \leq t \leq \beta .
$$

We first reduce the situation to the special case $0 \leq \alpha<\beta \leq 1$. In fact, for $\xi:=0 \wedge \alpha$ and $\eta:=1 \vee \beta$ we have $a:=(1-\xi) u+\xi v \in K$ and $b:=(1-\eta) u+\eta v \in K$, and

$$
(1-s) a+s b=(1-t) u+t v \quad \text { with } \quad t=(1-s) \xi+s \eta .
$$

It follows that both $\{(1-t) u+t v: 0 \leq t \leq 1\}$ and $\{(1-t) u+t v: \alpha \leq t \leq \beta\}$ are contained in $\{(1-t) u+t v: \xi \leq t \leq \eta\}=\{(1-s) a+s b: 0 \leq s \leq 1\}$. Thus for $a, b \in K$ we are in the special case, and the assertion for $a, b$ implies that for $u, v$.

(2) For $u, v \in K$ in the special case there are $0<\alpha<\beta<1$ such that

$$
f\left(\left(1-h_{t}(s)\right) u+h_{t}(s) v\right) \leq \text { some real } c \quad \text { for } \alpha \leq s \leq \beta \text { and for } s=0,1 .
$$

It remains to show that this relation holds for all $0 \leq s \leq 1$, that is, it remains true for $0<s<\alpha$ and $\beta<s<1$. We shall restrict ourselves to the 
case $0<s<\alpha$, and put $x=\left(1-h_{t}(s)\right) u+h_{t}(s) v$. We choose $n \in \mathbb{N}$ such that

$$
2^{n} s \frac{\beta-\alpha}{\alpha \beta}=2^{n} \frac{s}{\alpha}-2^{n} \frac{s}{\beta}>1 .
$$

Then there exists $k \in \mathbb{Z}$ with $2^{n} s / \beta<k<2^{n} s / \alpha$, so that $1 \leq k<2^{n}$. We put $\delta:=2^{n} s / k$ and obtain $0<s<\alpha<\delta<\beta<1$. Now we apply Part 2 to the pair $0<\delta$ with $u=\left(1-h_{t}(0)\right) u+h_{t}(0) v$ and $w:=\left(1-h_{t}(\delta)\right) u+h_{t}(\delta) v$, and to $0<s<\delta$ with $x=\left(1-h_{t}(s)\right) u+h_{t}(s) v$. It follows that $x=$ $\left(1-h_{\tau}(\sigma)\right) u+h_{\tau}(\sigma) w$ with $\sigma=\frac{s-0}{\delta-0}=\frac{s}{\delta}=k 2^{-n}$, which thus is dyadic rational. From $f(u), f(v) \leq c$ and Part 1 we therefore obtain $f(x) \leq c$.

PART 4. We have

$$
f\left(\left(1-h_{t}(s)\right) u+h_{t}(s) v\right) \leq\left(1-h_{t}(s)\right) f(u)+h_{t}(s) f(v) \quad \text { for } 0 \leq s \leq 1 .
$$

In view of 2.5(2) this is the final assertion.

Proof. (0) Assume that the assertion is not true. Then there exists $0<$ $s<1$ such that

$x=\left(1-h_{t}(s)\right) u+h_{t}(s) v \quad$ satisfies $\quad f(x)>\left(1-h_{t}(s)\right) f(u)+h_{t}(s) f(v)=: R$.

(1) In view of Part $1, s$ is not dyadic rational. Thus for each $n \in \mathbb{N}$ there exists $p(n) \in \mathbb{Z}$ such that $0 \leq 2^{-n}(p(n)-1)<s<2^{-n} p(n) \leq 1$; hence $1 \leq p(n) \leq 2^{n}$. We put $\alpha_{n}=2^{-n}(p(n)-1)$, so that

$$
0 \leq \alpha_{n}<s<\alpha_{n}+2^{-n} \leq 1 \text { and hence } 0<s-\alpha_{n}<2^{-n} .
$$

Then let $a_{n}:=\left(1-h_{t}\left(\alpha_{n}\right)\right) u+h_{t}\left(\alpha_{n}\right) v$. From Part 1 we have

$$
f\left(a_{n}\right) \leq\left(1-h_{t}\left(\alpha_{n}\right)\right) f(u)+h_{t}\left(\alpha_{n}\right) f(v) .
$$

(2) From $s<1$ we see that $\left(1-\alpha_{n}\right) /\left(s-\alpha_{n}\right)>1$. Now $\left(1-\alpha_{n}\right) /\left(s-\alpha_{n}\right)$ cannot be $2^{q}$ for $q \in \mathbb{N}$, because then $2^{q} s=1+\left(2^{q}-1\right) \alpha_{n}=1+2^{-n}\left(2^{q}-1\right)(p(n)-1) \quad$ and hence $2^{n+q} s \in \mathbb{N}$, so that $s$ would be dyadic rational. Thus there exists an integer $q(n) \geq 0$ such that $2^{q(n)}<\left(1-\alpha_{n}\right) /\left(s-\alpha_{n}\right)<2^{q(n)+1}$. From (1) it follows that

$$
2^{q(n)+1}>\frac{1-s}{s-\alpha_{n}}>2^{n}(1-s) \text { and hence } 2^{q(n)}>2^{n-1}(1-s) .
$$

Now we put $\beta_{n}=\alpha_{n}+2^{q(n)}\left(s-\alpha_{n}\right)$, so that

$$
2^{q(n)} s=\beta_{n}+\left(2^{q(n)}-1\right) \alpha_{n} \quad \text { or } \quad s=\left(1-2^{-q(n)}\right) \alpha_{n}+2^{-q(n)} \beta_{n} .
$$

It follows that $0 \leq \alpha_{n}<s \leq \beta_{n}<1$. We also set $b_{n}:=\left(1-h_{t}\left(\beta_{n}\right)\right) u+$ $h_{t}\left(\beta_{n}\right) v$.

(3) Now we apply Part 2 to the pair $\alpha_{n}<\beta_{n}$ with $a_{n}$ and $b_{n}$, and to $\alpha_{n}<s \leq \beta_{n}$ with $x$. It follows that

$$
x=\left(1-h_{\tau(n)}(\sigma(n))\right) a_{n}+h_{\tau(n)}(\sigma(n)) b_{n},
$$


where $\tau(n)=t\left(\beta_{n}-\alpha_{n}\right)$ and hence $|\tau(n)| \leq|t|$, and $\sigma(n)=\left(s-\alpha_{n}\right) /\left(\beta_{n}-\alpha_{n}\right)$ $=2^{-q(n)}$. Thus from Part 1 we obtain

$$
f(x) \leq\left(1-h_{\tau(n)}(\sigma(n))\right) f\left(a_{n}\right)+h_{\tau(n)}(\sigma(n)) f\left(b_{n}\right) .
$$

(4) For the final step we recall the last formulas of (0), (1) and (3) above. Moreover we see from 2.5(2) combined with (1)-(3) that

$$
\begin{aligned}
0<h_{t}(s)-h_{t}\left(\alpha_{n}\right) & \leq e^{|t|}\left(s-\alpha_{n}\right)<2^{-n} e^{|t|}, \\
0<h_{\tau(n)}(\sigma(n)) & \leq e^{|\tau(n)|} \sigma(n) \leq 2^{-q(n)} e^{|t|}<2^{-n} \frac{2}{1-s} e^{|t|} .
\end{aligned}
$$

It follows that

$$
\begin{aligned}
(1- & \left.h_{\tau(n)}(\sigma(n))\right)(f(x)-R) \\
= & f(x)-h_{\tau(n)}(\sigma(n)) f(x)-\left(1-h_{\tau(n)}(\sigma(n))\right)\left(R-f\left(a_{n}\right)\right) \\
& -\left(1-h_{\tau(n)}(\sigma(n))\right) f\left(a_{n}\right) \\
\leq & h_{\tau(n)}(\sigma(n)) f\left(b_{n}\right)-h_{\tau(n)}(\sigma(n)) f(x) \\
& -\left(1-h_{\tau(n)}(\sigma(n))\right)\left(h_{t}(s)-h_{t}\left(\alpha_{n}\right)\right)(f(v)-f(u)) \\
\leq & h_{\tau(n)}(\sigma(n)) c+h_{\tau(n)}(\sigma(n))|f(x)|+\left(h_{t}(s)-h_{t}\left(\alpha_{n}\right)\right)|f(v)-f(u)| \\
\leq & 2^{-n} e^{|t|}\left(\frac{2}{1-s}(c+|f(x)|)+|f(v)-f(u)|\right)
\end{aligned}
$$

with $0<c<\infty$ from Part 3. For $n \rightarrow \infty$ we obtain $f(x) \leq R$, which contradicts the assumption. This completes the proof of 2.4 .

The strengthened basic step and the second proof. The strengthened versions of the basic step 2.1 which result from 2.3 and 2.4 read as follows.

2.6. Proposition. Assume that $I:] 0, \infty\left[^{n} \rightarrow \mathbb{R}\right.$ is positive-homogeneous. Further assumptions are:

(1) for each pair $u, v \in] 0, \infty\left[^{n}\right.$ the function $t \mapsto I((1-t) u+t v)$ is continuous on $0<t<1$;

(2) for each pair $u, v \in] 0, \infty\left[^{n}\right.$ the function $t \mapsto I((1-t) u+t v)$ is bounded (above/below) on some nondegenerate subinterval of $\{t \in \mathbb{R}:(1-t) u+t v$ $>0\}$.

Of course $(1) \Rightarrow(2)$. Each of these assumptions implies that I satisfies the fundamental implication.

The point is that the efforts in order to obtain the fundamental implication from the two assumptions (1) and (2) are so different: Under (1) we shall use 2.3 , while under (2) we have to invoke 2.4 . This time the proof is 
by induction. The case $n=1$ is obvious. The induction step will be based on the lemma which follows.

2.7. Lemma. Assume that $I:] 0, \infty\left[{ }^{n+1} \rightarrow \mathbb{R}\right.$ is positive-homogeneous and (sub/super)modular $\vee \wedge$, and that for each $0<c<\infty$ the function ] $0, \infty\left[^{n} \rightarrow \mathbb{R}: x \mapsto I\left(c x_{1}, x\right)\right.$ is (sub/super)additive. Then the function $f:] 0, \infty\left[^{n} \rightarrow \mathbb{R}\right.$ defined by $f(x)=I(1, x)$ satisfies

$$
\left.f\left(\frac{\sqrt{v_{1}} u+\sqrt{u_{1}} v}{\sqrt{v_{1}}+\sqrt{u_{1}}}\right) \leq / \geq \frac{\sqrt{v_{1}} f(u)+\sqrt{u_{1}} f(v)}{\sqrt{v_{1}}+\sqrt{u_{1}}} \quad \text { for } u, v \in\right] 0, \infty\left[{ }^{n} .\right.
$$

Proof of 2.7. We shall represent the points $x=\left(x_{0}, x_{1}, \ldots, x_{n}\right) \in \mathbb{R}^{n+1}$ in the form $x=\left(x_{0}, x^{\prime}\right)$ with $x^{\prime}=\left(x_{1}, \ldots, x_{n}\right) \in \mathbb{R}^{n}$. Fix $\left.u, v \in\right] 0, \infty\left[^{n}\right.$ and define $a, b \in] 0, \infty\left[^{n+1}\right.$ by

$$
a=\sqrt{v_{1}}(1, u) \vee \sqrt{u_{1}}(1, v), \quad b=\sqrt{v_{1}}(1, u) \wedge \sqrt{u_{1}}(1, v) .
$$

Then

$$
\begin{array}{ll}
a_{0}=\sqrt{v_{1}} \vee \sqrt{u_{1}}, & a_{1}=\sqrt{u_{1} v_{1}}\left(\sqrt{u_{1}} \vee \sqrt{v_{1}}\right)=\sqrt{u_{1} v_{1}} a_{0}, \\
b_{0}=\sqrt{v_{1}} \wedge \sqrt{u_{1}}, & b_{1}=\sqrt{u_{1} v_{1}}\left(\sqrt{u_{1}} \wedge \sqrt{v_{1}}\right)=\sqrt{u_{1} v_{1}} b_{0} .
\end{array}
$$

Thus $a_{0} / a_{1}=b_{0} / b_{1}=: c$. Thus the assumption furnishes on the one hand

$$
\begin{gathered}
I(a+b)=I\left(c a_{1}+c b_{1}, a^{\prime}+b^{\prime}\right)=I\left(\left(c a_{1}, a^{\prime}\right)+\left(c b_{1}, b^{\prime}\right)\right) \\
\leq / \geq I\left(c a_{1}, a^{\prime}\right)+I\left(c b_{1}, b^{\prime}\right)=I(a)+I(b),
\end{gathered}
$$

and on the other hand

$$
I(a)+I(b) \leq / \geq I\left(\sqrt{v_{1}}(1, u)\right)+I\left(\sqrt{u_{1}}(1, v)\right)=\sqrt{v_{1}} f(u)+\sqrt{u_{1}} f(v) .
$$

Moreover we have

$$
\begin{aligned}
a+b & =\sqrt{v_{1}}(1, u)+\sqrt{u_{1}}(1, v)=\left(\sqrt{v_{1}}+\sqrt{u_{1}}\right)\left(1, \frac{\sqrt{v_{1}} u+\sqrt{u_{1}} v}{\sqrt{v_{1}}+\sqrt{u_{1}}}\right), \\
I(a+b) & =\left(\sqrt{v_{1}}+\sqrt{u_{1}}\right) f\left(\frac{\sqrt{v_{1}} u+\sqrt{u_{1}} v}{\sqrt{v_{1}}+\sqrt{u_{1}}}\right) .
\end{aligned}
$$

These formulas combine to give the assertion.

Proof of the induction step in 2.6. Let $I:] 0, \infty\left[{ }^{n+1}\right.$ be as assumed in 2.6 and (sub/super)modular $\vee \wedge$. For fixed $0<c<\infty$ define $J:] 0, \infty\left[{ }^{n} \rightarrow \mathbb{R}\right.$ by $J(x)=I\left(c x_{1}, x\right)$. It is obvious that $J$ inherits all assumptions from $I$, and hence by the induction hypothesis it is (sub/super)additive. Thus 2.7 ensures that 2.3 and 2.4 can be applied, in the respective cases, to the function $\pm f$ for $f:] 0, \infty\left[{ }^{n} \rightarrow \mathbb{R}\right.$ defined $f(x)=I(1, x)$. Therefore $f$ is convex/concave. Then we see that for $u, v \in] 0, \infty\left[^{n+1}\right.$, 


$$
\begin{aligned}
I(u+v) & =\left(u_{0}+v_{0}\right) I\left(1, \frac{u^{\prime}+v^{\prime}}{u_{0}+v_{0}}\right) \\
& =\left(u_{0}+v_{0}\right) f\left(\frac{u_{0}}{u_{0}+v_{0}}\left(\frac{u^{\prime}}{u_{0}}\right)+\frac{v_{0}}{u_{0}+v_{0}}\left(\frac{v^{\prime}}{v_{0}}\right)\right) \\
\leq & >u_{0} f\left(\frac{u^{\prime}}{u_{0}}\right)+v_{0} f\left(\frac{v^{\prime}}{v_{0}}\right)=u_{0} I\left(1, \frac{u^{\prime}}{u_{0}}\right)+v_{0} I\left(1, \frac{v^{\prime}}{v_{0}}\right) \\
& =I(u)+I(v) .
\end{aligned}
$$

This is the assertion.

Final results in the finite situation. The basic step 2.1 was for the open positive cone $] 0, \infty\left[^{n}\right.$, but it implies at once the identical result for the full positive cone $\left[0, \infty\left[{ }^{n}\right.\right.$ of $\mathbb{R}^{n}$.

2.8. Remark. Assume that $I:\left[0, \infty\left[^{n} \rightarrow \mathbb{R}\right.\right.$ is positive-homogeneous and continuous. Then it satisfies the fundamental implication.

However, this assertion is kind of a dead end, because it carries an unnatural restriction: For $I:\left[0, \infty\left[^{n} \rightarrow \mathbb{R}\right.\right.$ positive-homogeneous the properties (sub/super)additive mean convex/concave, and it is well known that such functions need not be continuous at the boundaries of their domains. An example is the function

$$
I:\left[0, \infty\left[^ { 2 } \rightarrow \left[0, \infty\left[\quad \text { with } I(x)=x_{1} \text { for } x_{2}>0 \text { and } I(x)=0 \text { for } x_{2}=0,\right.\right.\right.\right.
$$

which is positive-homogeneous, supermodular and superadditive, and moreover increasing. Thus we continue to extend the specialization 2.2 and the strengthened version 2.6, which will not produce such restrictions but will require a bit more work. Moreover we want to allow the function $I$ to attain the value $\infty$.

2.9. TheOREM. Assume that $I:\left[0, \infty\left[^{n} \rightarrow[0, \infty]\right.\right.$ is positive-homogeneous and increasing. Then I satisfies the fundamental implication.

2.10. Theorem. Assume that $I:[0, \infty[n \rightarrow]-\infty, \infty]$ is positive-homogeneous, and for $u \leq v$ in $\left[0, \infty\left[^{n}\right.\right.$ satisfies $I(u)=\infty \Rightarrow I(v)=\infty$. Further assumptions are:

(1) for all $u, v \in\left[0, \infty\left[^{n}\right.\right.$ with $I(u+v)<\infty$ the function $t \mapsto I((1-t) u$ $+t v)$ is continuous on $0<t<1$;

(2) for all $u, v \in\left[0, \infty\left[^{n}\right.\right.$ with $I(u+v)<\infty$ the function $t \mapsto$ $I((1-t) u+t v)$ is bounded (above/below) on some nondegenerate subinterval of $\{t \in \mathbb{R}:(1-t) u+t v \geq 0\}$.

Of course $(1) \Rightarrow(2)$. Each of these assumptions implies that I satisfies the fundamental implication. 
Theorem 2.9 will be the foundation for the treatment of the full situation in Section 3, while Theorem 2.10 is our final result in the finite situation. We want to obtain the two theorems with a simultaneous proof.

Proof of 2.9 and 2.10. First of all $I(0)=I(t 0)=t I(0)$ for $0<t<\infty$ implies that $I(0)=0$ or $I(0)=\infty$, and from $I(0)=\infty$ we have $I \equiv \infty$. Thus we can assume that $I(0)=0$. This in particular settles the case $n=1$ : We have $I(x)=c x$ for $x \in] 0, \infty[$ with $c=I(1) \in]-\infty, \infty]$, and hence $I$ is additive. Thus it remains to perform the induction step.

For this purpose we assume $I:\left[0, \infty\left[^{n+1} \rightarrow\right]-\infty, \infty\right]$ to be positivehomogeneous with $I(0)=0$ and to satisfy the further assumptions of one fixed situation out of the three under consideration, and moreover to be (sub/super)modular $\vee \wedge$. We define $J:\left[0, \infty\left[{ }^{n} \rightarrow\right]-\infty, \infty\right]$ by $J(x)=$ $I(0, x)$. It is obvious that $J$ inherits all assumptions from $I$, and hence by the induction hypothesis it is (sub/super)additive.

Then in all situations we have the alternative between

$$
\text { ( } I<\infty \text { and }(\star \star) I \mid] 0, \infty\left[^{n+1} \equiv \infty .\right.
$$

In fact, if $I(a)<\infty$ for some $a \in] 0, \infty\left[^{n+1}\right.$ then for each $x \in\left[0, \infty\left[{ }^{n+1}\right.\right.$ the relation $x \leq t a$ with some $0<t<\infty$ forces $I(x)<\infty$. Thus we have to prove that $I$ is (sub/super)additive both under $(\star)$ and $(\star \star)$.

First assume $(\star)$. In case 2.9 it follows from 2.2 that $I \mid] 0, \infty\left[^{n+1}\right.$ is (sub/super)additive. In case 2.10 the same follows from 2.6. It remains to consider $u, v \in\left[0, \infty\left[^{n+1}\right.\right.$ not both in $] 0, \infty\left[^{n+1}\right.$. We can assume that $u_{0}=0$, and use the notation $x=\left(x_{0}, x^{\prime}\right)$ as in the proof of 2.7 above. We have

$$
u+v=\left(0, u^{\prime}+v^{\prime}\right) \vee v \quad \text { and } \quad\left(0, v^{\prime}\right)=\left(0, u^{\prime}+v^{\prime}\right) \wedge v,
$$

which implies that

$$
\begin{aligned}
& I(u+v)+I\left(0, v^{\prime}\right) \leq / \geq I\left(0, u^{\prime}+v^{\prime}\right)+I(v)=J\left(u^{\prime}+v^{\prime}\right)+I(v) \\
& \leq / \geq J\left(u^{\prime}\right)+J\left(v^{\prime}\right)+I(v)=I\left(0, u^{\prime}\right)+I\left(0, v^{\prime}\right)+I(v)=I(u)+I(v)+I\left(0, v^{\prime}\right),
\end{aligned}
$$

and hence $I(u+v) \leq / \geq I(u)+I(v)$ since $I\left(0, v^{\prime}\right)<\infty$.

Now assume $(\star \star)$ and fix $u, v \in\left[0, \infty\left[^{n+1}\right.\right.$. There are two cases to consider:

(i) there exists $l \in\{0,1, \ldots, n\}$ with $u_{l}=v_{l}=0$,

(ii) for all $l \in\{0,1, \ldots, n\}$ we have $u_{l}+v_{l} \geq u_{l} \vee v_{l}>0$.

In case (i) we can assume that $u_{0}=v_{0}=0$. Then $I(u+v)=J\left(u^{\prime}+v^{\prime}\right) \leq / \geq$ $J\left(u^{\prime}\right)+J\left(v^{\prime}\right)=I(u)+I(v)$. In case (ii) we have $\left.u+v, u \vee v \in\right] 0, \infty\left[^{n+1}\right.$ and hence $I(u+v)=I(u \vee v)=\infty$. The relation $I(u \vee v)=\infty$ settles the sub case because it forces $I(u)+I(v)=\infty$, and $I(u+v)=\infty$ settles the super case. 
Finally, we transfer the results into the versions which follow. Let $X$ be a nonvoid set, and define $F(X) \subset\left[0, \infty\left[{ }^{X}\right.\right.$ to consist of the functions $f \in\left[0, \infty\left[{ }^{X}\right.\right.$ with finitely many values. Then $F(X)$ is a convex cone with $0 \in F(X)$ and stable under the pointwise lattice operations $\vee \wedge$.

2.11. Theorem. Assume that $I: F(X) \rightarrow[0, \infty]$ is positive-homogeneous and increasing. Then I satisfies the fundamental implication.

2.12. Theorem. Assume that $I: F(X) \rightarrow]-\infty, \infty]$ is positive-homogeneous, and for $u \leq v$ in $F(X)$ satisfies $I(u)=\infty \Rightarrow I(v)=\infty$. Further assumptions are:

(1) for all $u, v \in F(X)$ with $I(u+v)<\infty$ the function $t \mapsto I((1-t) u+t v)$ is continuous on $0<t<1$;

(2) for all $u, v \in F(X)$ with $I(u+v)<\infty$ the function $t \mapsto I((1-t) u+t v)$ is bounded (above/below) on some nondegenerate subinterval of $\{t \in \mathbb{R}$ : $(1-t) u+t v \geq 0\}$.

Of course $(1) \Rightarrow(2)$. Each of these assumptions implies that I satisfies the fundamental implication.

Proof of 2.11 and 2.12. Assume $I: F(X) \rightarrow]-\infty, \infty]$ to be positivehomogeneous and to satisfy the further assumptions of one of our three situations, and moreover to be (sub/super)modular $\vee \wedge$. Fix $u, v \in F(X)$ and let $X=X(1) \cup \ldots \cup X(n)$ be a decomposition of $X$ such that $u \mid X(l)=a_{l}$ and $v \mid X(l)=b_{l}$ for $1 \leq l \leq n$, and set $a=\left(a_{1}, \ldots, a_{n}\right)$ and $b=\left(b_{1}, \ldots, b_{n}\right)$ in $\left[0, \infty\left[{ }^{n}\right.\right.$. Define $\vartheta:\left[0, \infty\left[^{n} \rightarrow F(X)\right.\right.$ by

$$
x=\left(x_{1}, \ldots, x_{n}\right) \mapsto \vartheta(x)=\sum_{l=1}^{n} x_{l} \chi_{X(l)},
$$

so that $\vartheta(a)=u$ and $\vartheta(b)=v$. The map $\vartheta$ is positive-linear and increasing under $\leq$, and commutes with the pointwise $\vee \wedge$. Then define $J=I \circ \vartheta$ : $\left[0, \infty\left[^{n} \rightarrow\right]-\infty, \infty\right]$. It is obvious that $J$ inherits all assumptions from $I$, and hence is (sub/super)additive by 2.9 and 2.10. It follows that $I(u+v)=$ $I(\vartheta(a+b))=J(a+b) \leq / \geq J(a)+J(b)=I(\vartheta(a))+I(\vartheta(b))=I(u)+I(v)$.

\section{THE FULL SITUATION}

Return to the Choquet integral. We follow the model of the Choquet integral and have to recall some further of its properties. As above we refer to [7, Section 11] and [8, Section 2].

We need a few terms on nonvoid function systems $S \subset[0, \infty]^{X}$ and functionals $I: S \rightarrow[0, \infty]$. We define $S$ to be Stonean if $f \in S \Rightarrow$ $f \wedge t,(f-t)^{+} \in S$ for $0<t<\infty$; note that $f=f \wedge t+(f-t)^{+}$. In 
this case $I$ is called Stonean if

$$
I(f)=I(f \wedge t)+I\left((f-t)^{+}\right) \quad \text { for all } f \in S \text { and } 0<t<\infty .
$$

Moreover an increasing $I$ is called truncable if

$$
I(f)=\sup \left\{I\left((f-a)^{+} \wedge(b-a)\right): 0<a<b<\infty\right\} \quad \text { for all } f \in S .
$$

We note that this holds when $f$ on $[f>0]$ satisfies $\alpha \leq f \leq \beta$ for some constants $0<\alpha<\beta<\infty$, because then $f \leq(\alpha / \alpha-a)\left((f-a)^{+} \wedge(b-a)\right)$ for $0<a<\alpha<\beta<b<\infty$. Thus to be truncable is a mild continuity condition on $I$.

Next assume that $0 \in S$ and that $I$ is increasing with $I(0)=0$. Then we define the envelopes $I^{\star}, I_{\star}:[0, \infty]^{X} \rightarrow[0, \infty]$ by

$$
\begin{aligned}
& I^{\star}(f)=\inf \{I(u): u \in S \text { with } u \geq f\} \quad \text { with } \inf \emptyset:=\infty, \\
& I_{\star}(f)=\sup \{I(u): u \in S \text { with } u \leq f\} .
\end{aligned}
$$

Thus $I_{\star} \leq I^{\star}$ and $I^{\star}\left|S=I_{\star}\right| S=I$. Moreover $I^{\star}$ and $I_{\star}$ are increasing. If $S$ is stable under $\vee \wedge$ then to be submodular $\vee \wedge$ carries over from $I$ to $I^{\star}$, and to be supermodular $\vee \wedge$ carries over from $I$ to $I_{\star}$.

One then notes the properties which follow. The subsequent representation theorem is in essence due to Greco [4]. Let $\mathfrak{S}$ be a lattice of subsets in $X$ with $\emptyset \in \mathfrak{S}$.

3.1. Properties. (i) $\mathrm{UM}(\mathfrak{S})$ and $\operatorname{LM}(\mathfrak{S})$ are Stonean.

(ii) For $\varphi: \mathfrak{S} \rightarrow[0, \infty]$ increasing with $\varphi(\emptyset)=0$ the Choquet integral $I(f)=f f d \varphi$ on $\operatorname{UM}(\mathfrak{S}) / \operatorname{LM}(\mathfrak{S})$ is Stonean and truncable.

3.2. Theorem. Assume that $S \subset \mathrm{UM}(\mathfrak{S}) / \mathrm{LM}(\mathfrak{S})$ is positive-homogeneous with $0 \in S$ and Stonean, and that $I: S \rightarrow[0, \infty]$ with $I(0)=0$ is increasing. Then

there exist increasing set functions $\varphi: \mathfrak{S} \rightarrow[0, \infty]$ with $\varphi(\emptyset)=0$ which represent $I$, that is, $I(f)=f f d \varphi$ for all $f \in S$

iff $I$ is Stonean and truncable. In this case an increasing $\varphi: \mathfrak{S} \rightarrow[0, \infty]$ with $\varphi(\emptyset)=0$ represents $I$ iff $I_{\star}\left(\chi_{A}\right) \leq \varphi(A) \leq I^{\star}\left(\chi_{A}\right)$ for all $A \in \mathfrak{S}$.

At this point we return to the final aim of the present enterprise. We observe that the above representation theorem 3.2 allows us to formulate the crucial additivity property of the Choquet integral $I(f)=f f d \varphi$, that is, the implication $\Rightarrow$ in (A), exclusively in terms of the functional I without reference to the set function $\varphi$.

3.3. Remark. On a nonvoid set $X$ the following are equivalent.

(i) For each lattice $\mathfrak{S}$ in $X$ with $\emptyset \in \mathfrak{S}$ and each increasing $\varphi: \mathfrak{S} \rightarrow$ $[0, \infty]$ with $\varphi(\emptyset)=0$ one has the $\Rightarrow$ implication in $(\mathrm{A})$. 
(ii) For each positive-homogeneous $S \subset[0, \infty]^{X}$ with $0 \in S$ which is stable under $\vee \wedge$ and Stonean, and for each positive-homogeneous $I: S \rightarrow$ $[0, \infty]$ with $I(0)=0$ which is increasing, Stonean and truncable, one has the fundamental implication.

Proof. One obtains (ii) $\Rightarrow$ (i) as an immediate consequence of 1.2 and 3.1 combined with $\Rightarrow$ in $(\mathrm{M})$. To see $(\mathrm{i}) \Rightarrow(\mathrm{ii})$ one applies 3.2 to $I$ and $\mathfrak{S}=\mathfrak{P}(X)$, and takes

$$
\begin{array}{ll}
\varphi(A)=I^{\star}\left(\chi_{A}\right) \quad & \text { for } A \subset X \text { when } I \text { is submodular } \vee \wedge, \\
\varphi(A)=I_{\star}\left(\chi_{A}\right) & \text { for } A \subset X \text { when } I \text { is supermodular } \vee \wedge .
\end{array}
$$

Then $\varphi: \mathfrak{P}(X) \rightarrow[0, \infty]$ represents $I$, and is (sub/super)modular in view of the properties of the envelopes noted above. Thus (i) asserts that $f \mapsto f f d \varphi$ on $[0, \infty]^{X}$ is (sub/super)additive, and hence that $I$ is (sub/super)additive in the sense of our definition.

The new formulation 3.3(ii) of the $\Rightarrow$ implication in $(\mathrm{A})$ looks in fact like the theorem on the fundamental implication we are searching for. However, there are several additional conditions: Besides the almost familiar condition that $I$ be increasing there are the conditions that $I$ be Stonean and truncable (with the prerequisite that $S$ be Stonean). The condition to be truncable can be dismissed as a mild continuity assumption. However, the condition that $I$ be Stonean is a critical one, because it expresses that $I$ is additive in a certain partial sense, and thus collides with the conclusion. In fact, there are situations where the prospective theorem will be invoked in order to conclude that I is Stonean. A case in point will be described below. Therefore it is imperative that in a comprehensive version of the theorem like the desired one the assumption that $I$ be Stonean does not occur.

Now the fundamental fact is that the above reformulation 3.3(ii) holds true without the assumption that I be Stonean. This will be the main and final result of the present work. It is much more comprehensive than 3.3(ii).

\section{The main theorem}

3.4. TheOrem. Assume that the positive-homogeneous $S \subset[0, \infty]^{X}$ with $0 \in S$ is stable under $\vee \wedge$ and Stonean, and that the positive-homogeneous $I: S \rightarrow[0, \infty]$ with $I(0)=0$ is increasing and truncable. Then $I$ satisfies the fundamental implication.

The proof starts from the result 2.11 in the finite situation and proceeds via certain approximations which make essential use of the assumption that $I$ is increasing. An important intermediate step is the specialization $S=$ $[0, \infty]^{X}$. 
3.5. Specialization. Assume that the positive-homogeneous $I:[0, \infty]^{X}$ $\rightarrow[0, \infty]$ with $I(0)=0$ is increasing and truncable. Then $I$ satisfies the fundamental implication.

The above specialization is the first 1998 result of the author in the present context [8, Theorem 1.1]. It is, aside from the theories of measure and integration developed in [7], the basic pillar which carries the comprehensive Daniell-Stone and Riesz type representation theorems $[8,5.3]=[9,6.3]$ and $[8,5.8]=[9,6.6]$. These theorems are the other important application of the new (sub/super)additivity theorem. They will be described in the final subsection below. We note that the applications of $[8,1.1]$ which served to obtain these theorems were in the proof of $[8,3.10]$ and had in fact the aim to prove that the functionals under consideration were Stonean. For the details we have to refer to that paper.

Proof of 3.5. (0) We first recall the basic estimate 11.6 from the author's textbook [7]. We use the $\geq$ version, but the $>$ version would do as well. For $f: X \rightarrow \overline{\mathbb{R}}$ and real $a=t(0)<t(1)<\ldots<t(r)=b$ the estimate reads

$$
\sum_{l=1}^{r}(t(l)-t(l-1)) \chi_{[f \geq t(l)]} \leq(f-a)^{+} \wedge(b-a) \leq \sum_{l=1}^{r}(t(l)-t(l-1)) \chi_{[f \geq t(l-1)]} .
$$

We also note for $\delta:=\max \{t(l)-t(l-1): 1 \leq l \leq r\}$ the estimate

$$
\begin{aligned}
\sum_{l=1}^{r}(t(l)-t(l-1)) \chi_{[f \geq t(l-1)]} & =\sum_{l=1}^{r}(t(l)-t(l-1))\left(\chi_{[f \geq t(l)]}+\chi_{[t(l-1) \leq f<t(l)]}\right) \\
& \leq \sum_{l=1}^{r}(t(l)-t(l-1)) \chi_{[f \geq t(l)]}+\delta \chi_{[a \leq f<b]} .
\end{aligned}
$$

(1) We prove the sub implication. Assume that $I$ is submodular $\vee \wedge$ and fix $u, v \in[0, \infty]^{X}$. We can assume that $I(u), I(v)<\infty$. For $0<a, c<\infty$ let $b=a+c$. For $a=t(0)<t(1)<\ldots<t(r)=b$ we see from (0) that

$$
\begin{aligned}
((u+v)-2 a)^{+} \wedge c= & ((u-a)+(v-a))^{+} \wedge c \\
\leq & \left((u-a)^{+}+(v-a)^{+}\right) \wedge c \leq(u-a)^{+} \wedge c+(v-a)^{+} \wedge c \\
\leq & \sum_{l=1}^{r}(t(l)-t(l-1)) \chi_{[u \geq t(l)]}+\delta \chi_{[a \leq u<b]} \\
& +\sum_{l=1}^{r}(t(l)-t(l-1)) \chi_{[v \geq t(l)]}+\delta \chi_{[a \leq v<b]} .
\end{aligned}
$$

We know from 2.11 that $I \mid F(X)$ is subadditive. From this fact and once more from the first estimate in $(0)$ we obtain 


$$
\begin{aligned}
I\left(((u+v)-2 a)^{+} \wedge c\right) \leq & I\left(\sum_{l=1}^{r}(t(l)-t(l-1)) \chi_{[u \geq t(l)]}\right)+\delta I\left(\chi_{[a \leq u<b]}\right) \\
& +I\left(\sum_{l=1}^{r}(t(l)-t(l-1)) \chi_{[v \geq t(l)]}\right)+\delta I\left(\chi_{[a \leq v<b]}\right) \\
\leq & \left(1+\frac{\delta}{a}\right) I(u)+\left(1+\frac{\delta}{a}\right) I(v) .
\end{aligned}
$$

It follows that

$$
I\left(((u+v)-2 a)^{+} \wedge c\right) \leq I(u)+I(v) \text { for all } 0<a, c<\infty,
$$

and hence $I(u+v) \leq I(u)+I(v)$ since $I$ is truncable.

(2) We prove the super implication. Assume that $I$ is supermodular $\vee \wedge$ and fix $u, v \in[0, \infty]^{X}$. We can assume that $I(u+v)<\infty$. For $0<a<b<\infty$ and $a=t(0)<t(1)<\ldots<t(r)=b$ we see from $(0)$ that

$$
\begin{aligned}
(1+\delta / a)(u+v) \geq & (u-a)^{+} \wedge(b-a)+\delta \chi_{[a \leq u<b]} \\
& +(v-a)^{+} \wedge(b-a)+\delta \chi_{[a \leq v<b]} \\
\geq & \sum_{l=1}^{r}(t(l)-t(l-1)) \chi_{[u \geq t(l-1)]} \\
& +\sum_{l=1}^{r}(t(l)-t(l-1)) \chi_{[v \geq t(l-1)]} .
\end{aligned}
$$

As before we know from 2.11 that $I \mid F(X)$ is superadditive. From this fact and once more from the first estimate in $(0)$ we obtain

$$
\begin{aligned}
(1+\delta / a) I(u+v) \geq & I\left(\sum_{l=1}^{r}(t(l)-t(l-1)) \chi_{[u \geq t(l-1)]}\right) \\
& +I\left(\sum_{l=1}^{r}(t(l)-t(l-1)) \chi_{[v \geq t(l-1)]}\right) \\
\geq & I\left((u-a)^{+} \wedge(b-a)\right)+I\left((v-a)^{+} \wedge(b-a)\right) .
\end{aligned}
$$

It follows that

$$
I(u+v) \geq I\left((u-a)^{+} \wedge(b-a)\right)+I\left((v-a)^{+} \wedge(b-a)\right)
$$

for all $0<a<b<\infty$, and hence $I(u+v) \geq I(u)+I(v)$ since $I$ is truncable.

Proof of 3.4. (1) The super implication is a simple application of 3.5. Assume that $I$ is supermodular $\vee \wedge$.

(1.1) We know that the envelope $I_{\star}:[0, \infty]^{X} \rightarrow[0, \infty]$ is positive-homogeneous, increasing and supermodular $\vee \wedge$. We claim that $I_{\star}$ is truncable as well. To see this fix $f \in[0, \infty]^{X}$ and $c<I_{\star}(f)$, and then $u \in S$ with $u \leq f$ 
and $c<I(u)$. Since $S$ is Stonean and $I$ is truncable there exist $0<a<b<\infty$ such that $c<I\left((u-a)^{+} \wedge(b-a)\right)$. It follows that $c<I_{\star}\left((f-a)^{+} \wedge(b-a)\right)$, which proves the claim.

(1.2) We conclude from 3.5 that $I_{\star}$ is superadditive. In view of our definition therefore $I_{\star} \mid S=I$ is superadditive as well.

(2) The sub implication is more involved. Assume that $I$ is submodular $\vee \wedge$.

(2.1) We know that the envelope $I^{\star}:[0, \infty]^{X} \rightarrow[0, \infty]$ is positive-homogeneous, increasing and submodular $\vee \wedge$. But we cannot assert that $I^{\star}$ is truncable.

(2.2) In order to proceed we consider for fixed $0<a<b<\infty$ the map $[0, \infty] \rightarrow[0, \infty]$ defined by $x \mapsto x_{a}^{b}:=(x-a)^{+} \wedge(b-a)$. Its relevant properties are as follows.

(i) $(t x)_{t a}^{t b}=t\left(x_{a}^{b}\right)$ for $0<t<\infty$.

(ii) The map $x \mapsto x_{a}^{b}$ is increasing.

(iii) $x_{a}^{b}$ is decreasing in $a$ and increasing in $b$.

(iv) $(u \vee v)_{a}^{b}=u_{a}^{b} \vee v_{a}^{b}$ and $(u \wedge v)_{a}^{b}=u_{a}^{b} \wedge v_{a}^{b}$.

(v) For $0<s<a<b<\infty$ we have $x_{a}^{b}=\left(x_{s}^{b}\right)_{a-s}^{b-s}$.

Here (i)-(iii) are obvious, and (iv) is an immediate consequence of (ii). To see (v) one distinguishes three cases: $x \in[0, a],[a, b],[b, \infty]$.

(2.3) Then we define the functional $J:[0, \infty]^{X} \rightarrow[0, \infty]$ by

$$
J(f)=\sup \left\{I^{\star}\left(f_{a}^{b}\right): 0<a<b<\infty\right\} \quad \text { with } \quad f_{a}^{b}:=(f-a)^{+} \wedge(b-a) .
$$

Then first of all $J \mid S=I$, because $S$ is Stonean, $I^{\star} \mid S=I$ and $I$ is truncable. Next one concludes from (i) that $J$ is positive-homogeneous, from (ii) that $J$ is increasing, and from (iii)-(iv) that $J$ is submodular $\vee \wedge$. We claim that $J$ is truncable as well. To see this fix $f \in[0, \infty]^{X}, c<J(f)$, and $0<a<b<\infty$ such that $c<I^{\star}\left(f_{a}^{b}\right)$. For $0<s<a<b<\infty$ we deduce from (v) that $c<I^{\star}\left(\left(f_{s}^{b}\right)_{a-s}^{b-s}\right) \leq J\left(f_{s}^{b}\right)$, which proves the claim.

(2.4) We conclude from 3.5 that $J$ is subadditive. In view of our definition, $J \mid S=I$ is therefore subadditive as well. This finishes the proof of 3.4 .

Another example. We want to add one more example, in order to show what can happen when the functional $I$ is not increasing.

3.6. Example. Let $X \subset \mathbb{R}$ be an interval with $\sup X=\infty$. Define $P \subset\left[0, \infty\left[{ }^{X}\right.\right.$ to consist of the functions $f: X \rightarrow[0, \infty[$ which are constant near $\infty$, that is, on some upward unbounded subinterval of $X$, and $Q \subset\left[0, \infty\left[{ }^{X}\right.\right.$ to consist of the functions $f: X \rightarrow[0, \infty[$ which are strictly decreasing near $\infty$. Then $P \cap Q=\emptyset$, and $S:=P \cup Q \subset\left[0, \infty\left[^{X}\right.\right.$ is a convex cone 
with $0 \in S$ which is stable under $\vee \wedge$. Define $I: S \rightarrow[0, \infty[$ by $I(f)=0$ for $f \in P$ and $I(f)=\lim _{t \rightarrow \infty} f(t)$ for $f \in Q$. Then $I$ is positive-homogeneous with $I(0)=0$, but of course not increasing. One verifies that $I$ is modular $\vee \wedge$. But $I$ is not additive, since for $u \in P$ with $u=c>0$ near $\infty$ and $v \in Q$ one has $u+v \in Q$ with $I(u+v)=c+I(v)>I(v)=I(u)+I(v)$. We note that $I$ has certain continuity properties. Thus $S$ is Stonean, and $I$ is truncable in the sense that

$$
I\left((f-a)^{+} \wedge(b-a)\right) \uparrow I(f) \text { as } a \downarrow 0 \text { and } b \uparrow \infty \quad \text { for all } f \in S .
$$

Also for each pair $u, v \in S$ the function $t \mapsto I((1-t) u+t v)$ is continuous on $0<t<1$ (but need not be continuous on $0 \leq t \leq 1$ ).

The Daniell-Stone-Riesz representation theorems. The representation theorems of the present subsection are quite different from the former representation theorem 3.2: The aim is to represent particular classes of functionals in terms of certain classes of distinguished set functions, as in the classical Riesz representation theorem, but in a much more extended setting. The basis is the extension theories in measure and integration developed in the author's textbook [7] and in subsequent articles like [8], and summarized in [9]. We recall that there are parallel inner and outer extension theories, and also parallel sequential and nonsequential versions (as usual labelled as $\sigma$ and $\tau$ versions). We also recall the most basic notions: For an increasing set function $\varphi: \mathfrak{S} \rightarrow[0, \infty]$ on a set system $\mathfrak{S}$ with $\emptyset \in \mathfrak{S}$ and $\varphi(\emptyset)=0$ and for $\bullet=\sigma, \tau$ one forms the envelopes

$\varphi_{\bullet}, \varphi^{\bullet}: \mathfrak{P}(X) \rightarrow[0, \infty]$ with the satellites $\varphi_{\bullet}^{B}: \mathfrak{P}(X) \rightarrow[0, \infty[$ with $B \in \mathfrak{S}$, and on a lattice $\mathfrak{S}$ with $\emptyset \in \mathfrak{S}$ one defines the inner and outer $\bullet$-premeasures $\varphi$. Likewise for an increasing functional $I: S \rightarrow[0, \infty]$ on a function class $S \subset[0, \infty]^{X}$ with $0 \in S$ and $I(0)=0$ and for $\bullet=\sigma, \tau$ one forms the envelopes

$I_{\bullet}, I^{\bullet}:[0, \infty]^{X} \rightarrow[0, \infty]$ with the satellites $I_{\bullet}^{v}:[0, \infty]^{X} \rightarrow[0, \infty]$ with $v \in S$; the notions of inner and outer •-preintegrals $I$ will appear below.

From now on, we fix a positive-homogeneous function class

$$
\begin{aligned}
& S \subset[0, \infty]^{X} \quad \text { in the inner situation, } \\
& S \subset[0, \infty]^{X} \quad \text { in the outer situation, }
\end{aligned}
$$

with $0 \in S$ which is stable under $\vee \wedge$ and Stonean, and a functional

$$
\begin{aligned}
& I: S \rightarrow[0, \infty[\quad \text { in the inner situation, } \\
& I: S \rightarrow[0, \infty] \quad \text { in the outer situation, }
\end{aligned}
$$

with $I(0)=0$ which is increasing. These are the assumptions made in [8], [9], 
while those in [7] were much narrower. We form the set systems $\operatorname{um}(S)=\{[f \geq t]: f \in S$ and $0<t<\infty\} \quad$ for the inner situation, $\operatorname{lm}(S)=\{[f>t]: f \in S$ and $0<t<\infty\} \quad$ for the outer situation,

which are lattices with $\emptyset$. Then we define

- the inner sources of $I$ to be those increasing set functions $\varphi: \operatorname{um}(S) \rightarrow[0, \infty[$,

- the outer sources of $I$ to be those increasing set functions $\varphi: \operatorname{lm}(S) \rightarrow[0, \infty]$,

which have $\varphi(\emptyset)=0$ and which represent $I, I(f)=f f d \varphi$ for all $f \in S$. The representation Theorem 3.2 tells us that such inner/outer sources of $I$ exist iff $I$ is Stonean and truncable. In this case their characterization is $I_{\star}\left(\chi_{A}\right) \leq \varphi(A) \leq I^{\star}\left(\chi_{A}\right)$ for all $A \in \mathrm{um}(S) / \operatorname{lm}(S)$, so that as a rule one must expect a lot of inner and outer sources of $I$.

Next we define for $\bullet=\sigma, \tau$ the functional $I$ to be an inner/outer --preintegral if it admits at least one inner/outer source which is an inner/outer •-premeasure. Then the fundamental results quoted above are the theorems on the inner and outer $\bullet$ preintegrals which follow.

3.7. Inner Theorem $(\bullet=\sigma, \tau)$. The functional $I$ is an inner $\bullet$-preintegral iff

(1) I is supermodular, Stonean and downward $\bullet$-continuous at $\emptyset$,

(2) $I(v) \leq I(u)+I_{\bullet}^{v}(v-u)$ for all $u \leq v$ in $S$.

In this case $\varphi:=I^{\star}(\chi) \mid. \operatorname{um}(S)$ is the unique inner source of $I$ which is an inner •-premeasure. It satisfies $I_{\bullet}(f)=f f d \varphi \bullet$ for all $f \in[0, \infty]^{X}$.

3.8. Outer Theorem $(\bullet=\sigma, \tau)$. The functional $I$ is an outer $\bullet$-preintegral iff

(1) $I$ is submodular, Stonean and upward $\bullet$-continuous,

(2) $I(v) \geq I(u)+I^{\bullet}(v-u)$ for all $u \leq v$ in $S$ with $u<\infty$,

(3) moreover for $\bullet=\tau$ (while this is automatic for $\bullet=\sigma$ )

$$
I^{\bullet}(f)=\sup \left\{I^{\bullet}(f \wedge u): u \in[I<\infty]\right\} \quad \text { for all } f \in\left[I^{\bullet}<\infty\right] .
$$

In this case $\varphi:=I_{\star}(\chi) \mid. \operatorname{lm}(S)$ is the unique outer source of $I$ which is an outer $\bullet$-premeasure. It satisfies $I^{\bullet}(f)=f f d \varphi^{\bullet}$ for all $f \in[0, \infty]^{X}$.

We refer to the cited papers for the collection of more or less familiar special cases. Thus the classical Riesz representation theorem and its extension to arbitrary Hausdorff topological spaces are immediate consequences of the inner $\tau$-theorem, whereas the conventional Daniell-Stone theorem falls under the outer $\sigma$-theorem. 


\section{References}

[1] R. C. Bassanezi and G. H. Greco, Sull'additività dell'integrale, Rend. Sem. Mat. Univ. Padova 72 (1984), 249-275.

[2] G. Choquet, Theory of capacities, Ann. Inst. Fourier (Grenoble) 5 (1953/54), 131295.

[3] D. Denneberg, Non-Additive Measure and Integration, Kluwer, 1994.

[4] G. H. Greco, Sulla rappresentazione di funzionali mediante integrali, Rend. Sem. Mat. Univ. Padova 66 (1982), 21-42.

[5] G. H. Hardy, J. E. Littlewood and G. Pólya, Inequalities, 2nd ed., Cambridge Univ. Press, 1952.

[6] J. Kindler, A Mazur-Orlicz theorem for submodular set functions, J. Math. Anal. Appl. 120 (1986), 533-564.

[7] H. König, Measure and Integration: An Advanced Course in Basic Procedures and Applications, Springer, 1997.

[8] - Measure and integration: Integral representations of isotone functionals, Ann. Univ. Sarav. Ser. Math. 9 (1998), 123-153.

[9] H. König, Measure and integration: An attempt at unified systematization, Rend. Istit. Mat. Univ. Trieste, to appear. Preprint under http://www.math.uni-sb.de/EX/ koenig/.

[10] H. Lebesgue, Sur une généralisation de l'intégrale définie, C. R. Acad. Sci. Paris 132 (1901), 1025-1027 and 332 (2001), 85-90.

[11] D. Schmeidler, Integral representation without additivity, Proc. Amer. Math. Soc. 97 (1986), 255-261.

[12] F. Topsøe, On construction of measures, in: Proc. Conf. Topology and Measure I (Zinnowitz, 1974), Part 2, Ernst-Moritz-Arndt Univ., Greifswald, 1978, 343-381.

Fakultät für Mathematik und Informatik

Universität des Saarlandes

D-66041 Saarbrücken, Germany

E-mail: hkoenig@math.uni-sb.de

Received June 12, 2002

Revised version March 25, 2003 